\title{
LCM-STABILITY OF POWER SERIES EXTENSIONS CHARACTERIZES DEDEKIND DOMAINS
}

\author{
JOHN T. CONDO
}

(Communicated by Louis J. Ratliff)

\begin{abstract}
In this paper we prove the following main result. A (commutative integral) domain $R$ is a Dedekind domain if and only if $R[[X]] \subset T[[X]]$ is LCM-stable for each domain $T$ containing $R$ as a subring.
\end{abstract}

\section{INTRODUCTION}

Suppose $R$ is a (commutative) domain with quotient field $K$. A result of F. Richman [12, Theorem 4] asserts that $R$ is a Prüfer domain if and only if each overring of $R$ (i.e., each $R$-subalgebra of $K$ ) is a $R$-flat. As a consequence, it is easy to see that $R$ is a Prüfer domain if and only if each domain $T$ containing $R$ as a subring induces a flat extension, $T[X]$ over $R[X]$, of polynomial rings. In fact, there is an ideal-theoretic variant in terms of the LCM-stability concept. (As in [8], [9], we say an $R$-module $M$ is LCM-stable if $a M \cap b M=(a R \cap b R) M$ for all $a, b \in R$.) Namely, $R$ is Prüfer is and only if $T[X]$ is LCM-stable over $R[X]$ for each domain $T$ containing $R$ as a subring. Our main interest here is to study the analogous property for power series rings. In other words, we seek to characterize domains $R$ such that $T[[x]]$ is LCM-stable over $R[[X]]$ for each domain $T$ containing $R$ as a subring. Our main result, Theorem 2.11 , is that these $R$ are precisely the Dedekind domains.

It is interesting to record additional motivation for the above problem. Since flatness implies LCM-stability and flatness is a universal property, a flat extension $R \subset T$ of domains entails LCM-stability of $R[X] \subset T[X]$. It is natural to ask whether LCM-stability of $R \subset T$ also entails LCM-stability of $R[X] \subset T[X]$. Several papers ([13], [15], [16]) have recently culminated in an affirmative answer [15, Corollary 3.7] in case $R$ is a GCD-domain; later work gave an affirmative answer in case $R$ is a Krull domain [16, Theorem 11]. These results naturally lead one to ask when LCM-stability of $R \subset T$ entails LCM-stability of $R[[X]] \subset T[[X]]$. The easiest case arises when all such $T$ are LCM-stable over $R$; and, by the remarks of the first paragraph, this happens

Received by the editors February 26, 1991 and, in revised form, November 23, 1993.

1991 Mathematics Subject Classification. Primary 13G05, 13F05, 13F25; Secondary 13C11, 13C15, 13B99, 13A18, 13F15.

This paper forms part of the author's Ph.D. dissertation written under the direction of Professor David E. Dobbs at the University of Tennessee. The author wishes to thank Professor Dobbs for his guidance and support. 
precisely for Prüfer domains $R$. To a large extent, the details of $\S 2$ amount to determining that the Dedekind domains are precisely the (Prüfer) domains with the property $R[[X]] \subset T[[X]]$ is LCM-stable for each $T$ containing $R$ as a subring.

Theorem 2.5 establishes that if $R$ is a Dedekind domain, then $T[[X]]$ is LCM-stable over $R[[X]]$ for each domain $T$ containing $R$ as a subring. With respect to the converse, namely determining which domains $R$ satisfy $T[[X]]$ is LCM-stable over $R[[X]]$ for all domains $T$ containing $R$ as a subring, Corollary 2.2 reduces the study to a class of Prüfer domains. By considering the non-Archimedean domains (in the sense of [14]), we reduce the problem to studying one-dimensional Prüfer domains in Corollary 2.7. The local case leads to DVR's in Theorem 2.8, thanks to an appeal to work of M. Anderson and J. Watkins [1, Lemma, p. 191]. The route back from DVR's to the (global) answer is essayed by means of a globalization result and appeals to work on almost Dedekind domains (in the sense of [10]) due to Arnold [2], [4], and to Arnold-Brewer [3].

A closing remark presents partial results on related analogous questions about flatness.

Throughout, given a symbol representing a power series, the individual coefficients are denoted by subscripting the symbol. For example, if $\alpha \in R[[X]]$, then $\alpha=\sum_{i=0}^{\infty} \alpha_{i} X^{i}$. If $\left\{\alpha_{j}\right\}_{j=1}^{n}$ is a collection of power series, we write $\alpha_{j}=\sum_{i=0}^{\infty} \alpha_{j, i} X^{i}$ for each $j$.

\section{Results}

Our goal is to classify the domains $R$ such that $R[[X]] \subset T[[X]]$ is LCMstable for all domains $T$ containing $R$ as a subring. We begin by showing in Proposition 2.2 that any such domain $R$ must be a Prüfer domain. We first state a lemma, whose computational proof is left to the reader.

Lemma 2.1. Suppose $R$ is a subring of a domain $S$. If $R[[X]] \subset S[[X]]$ is LCM-stable, then $R \subset S$ is LCM-stable.

Proposition 2.2. Let $R$ be a domain. If $R[[X]] \subset T[[X]]$ is LCM-stable for each domain $T$ containing $R$ as a subring, then $R$ is a Prüfer domain.

Proof. Lemma 2.1 shows $R \subset T$ is LCM-stable for all domains $T$ containing $R$ as a subring. In particular, $R \subset T$ is LCM-stable for all overrings $T$ of $R$. By a result of Uda [15, Proposition 1.7] $R \subset T$ is flat for all overrings $T$ of $R$. Hence $R$ is a Prüfer domain, by the earlier-cited result of F. Richman [12, Theorem 4].

We shall soon show that among the Prüfer domains, (at least) the Dedekind domains have the desired property. First, we prove two lemmas.

Lemma 2.3. Let $R$ be a UFD and let $T$ be a domain containing $R$ as a subring. Then $R \subset T$ is LCM-stable if (and only if) $a T \cap b T=(a R \cap b R) T$ for all $a, b \in R$ such that g.c.d. $(a, b)=1$.

Proof. The "only if" assertion is trivial. For the converse, let $a, b \in R$ and set $d=$ g.c.d. $(a, b)$. Put $a_{1}=a d^{-1}, b_{1}=b d^{-1}$; then g.c.d. $\left(a_{1}, b_{1}\right)=1$.

$a T \cap b T=d a_{1} T \cap d b_{1} T=d\left(a_{1} T \cap b_{1} T\right)$. Since g.c.d. $\left(a_{1}, b_{1}\right)=1, a_{1} T \cap$ 
$b_{1} T=\left(a_{1} R \cap b_{1} R\right) T$ by hypothesis. Therefore,

$$
a T \cap b T=d\left(\left(a_{1} R \cap b_{1} R\right) T\right)=\left(d a_{1} R \cap d b_{1} R\right) T=(a R \cap b R) T .
$$

Lemma 2.4. Suppose $R$ and $T$ are domains, with $R$ a subring of $T$. Let $S$ be a multiplicatively closed subset of $R[[X]]$ not containing $X$. Then $R[[X]]_{S} \subset$ $T[[X]]_{S}$ is LCM-stable if (and only if)

$$
f T[[X]]_{S} \cap g T[[X]]_{S}=\left(f R[[X]]_{S} \cap g R[[X]]_{S}\right) T[[X]]_{S}
$$

for all $f, g \in R[[X]]$ such that $f_{0} \neq 0$ and $g_{0} \neq 0$.

Proof. Once again, the "only if" assertion is trivial. For the converse, note that a typical element of $R[[X]]_{S}$ is of the form $r s^{-1}$ where $r \in R[[X]]$ and $s \in S$. Since $s$ is a unit of $R[[X]]_{S}, r R[[X]]_{S}=r s^{-1} R[[X]]_{S}$. Hence, to establish LCM-stability, it suffices to consider nonzero elements $f, g \in R[[X]]$. Then there exist integers $n, m \geq 0$ and $f^{*}, g^{*} \in R[[x]]$ with $f_{0}^{*} \neq 0$ and $g_{0}^{*} \neq 0$ such that $f=X^{n} f^{*}$ and $g=X^{m} g^{*}$. Now, suppose $\gamma \in f T[[X]]_{S} \cap g T[[X]]_{S}$. Then there exist $\alpha, \beta \in T[[X]]_{S}$ with $\gamma=f \alpha=g \beta$. So $X^{n} f^{*} \alpha=X^{m} g^{*} \beta$. Without loss of generality, $n \geq m$. Thus $X^{n-m} f^{*} \alpha=g^{*} \beta$. Since $X$ is prime in $T[[X]]_{S}$ and $X$ does not divide $g^{*}, X^{n-m}$ divides $\beta$. So there exists $\beta^{*} \in T[[X]]_{S}$ such that $\beta=X^{n-m} \beta^{*}$. Hence, $\gamma X^{-n}=f^{*} \alpha=g^{*} \beta$. By assumption, there exist $r_{j} \in f^{*} R[[X]]_{S} \cap g^{*} R[[X]]_{S}$ and $\tau_{j} \in T[[x]]_{S} \quad(1 \leq$ $j \leq n)$ with $\gamma X^{-n}=\sum_{j=1}^{n} r_{j} \tau_{j}$. Therefore, $\gamma=\sum_{j=1}^{n}\left(r_{j} X^{n}\right) \tau_{j}$. Since $r_{j} X^{n} \in$ $f R[[X]]_{S} \cap g R[[X]]_{S}$ for $1 \leq j \leq n, R[[X]]_{S} \subset T[[X]]_{S}$ is LCM-stable.

Theorem 2.5. Let $D$ be a Dedekind domain. Then $D[[X]] \subset T[[X]]$ is LCMstable for every domain $T$ containing $D$ as a subring.

Proof. By a result of Uda [15, Proposition 1.6], it is enough to prove that $D[[X]]_{(P, X)} \subset T[[x]]_{(P, X)}$ is LCM-stable for every nonzero prime ideal $P$ of $D$. Fix a prime ideal $P$, and set $R=D[[X]]_{(P, X)}$ and $S=T[[X]]_{(P, X)}$.

Since $D$ is regular, so is $D[[X]]$ (cf. [11, Exercise 5, p. 121]) and hence $R$ is regular local. Hence, a theorem of Auslander-Buchsbaum shows $R$ is a UFD (cf. [11, Theorem 184]). So, by Lemma 2.3, it is enough to show $f S \cap g S=(f R \cap g R) S$ for all $f, g \in R$ such that g.c.d. $(f, g)=1$. By Lemma 2.4, we assume $f, g \in D[[X]]$ and $f_{0} \neq 0$ and $g_{0} \neq 0$.

Finally, we may assume that neither $f$ nor $g$ is a unit of $R$. To see this, note that if $f$, for example, is a unit of $R$, then $f R=R$ and $f S=S$, whence $f S \cap g S=g S=(g R) S=(f R \cap g R) S$.

This last assumption shows $(f, g) \subset(P, X)_{(P, X)}$, the maximal ideal of $R$. Since $R$ is a UFD, the height 1 prime ideals of $R$ are principal. Then, g.c.d. $(f, g)=1$ and $\operatorname{dim} R=2$ show that $(P, X)_{(P, X)}$ is the only prime ideal of $R$ containing $(f, g)$. Therefore $\operatorname{rad}(f, g)=(P, X)_{(P, X)}$. Hence, there exist $r_{1}, r_{2} \in R$ and an integer $n \geq 1$ such that

$$
X^{n}=r_{1} f+r_{2} g \text {. }
$$

Consider $\gamma \in f S \cap g S$. There exist $\alpha, \beta \in S$ such that $\gamma=f \alpha=g \beta$. Multiplying both sides of the displayed equation by $\alpha$ yields $X^{n} \alpha=r_{1}(f \alpha)+$ $r_{2} g \alpha=r_{1}(g \beta)+r_{2} g \alpha=g\left(r_{1} \beta+r_{2} \alpha\right)$. Now $X$ is prime in $S$ and $g_{0} \neq 0$. Therefore, $\delta=\left(r_{1} \beta+r_{2} \alpha\right) X^{-n} \in S$. Then $\alpha=g \delta$ and $\gamma=f \alpha=f g \delta \in$ $(f R \cap g R) S$, so that $f S \cap g S=(f R \cap g R) S$. 
Results (2.6)-(2.10) will show that a Dedekind domain is the only type of (Prüfer) domain $R$ having the property that $R[[X]] \subset T[[X]]$ is LCM-stable for each domain $T$ containing $R$ as a subring. The next proposition will be used to prove that no Prüfer domain $R$ with $\operatorname{dim} R \geq 2$ can have this property. First, recall from [14] that a domain $R$ is Archimedean if $\bigcap_{n=1}^{\infty} d^{n} R=0$ for every nonzero nonunit $d \in R$; and recall (cf. [10]) that a valuation domain $V$ is a Archimedean if and only if $\operatorname{dim} V \leq 1$.

Proposition 2.6. Let $R$ be a non-Archimedean domain. Then there exists an $R$-flat domain $T$ containing $R$ as a subring such that $R[[X]] \subset T[[X]]$ is not LCM-stable; it can be arranged that $T$ is an overring of $R$.

Proof. Since $R$ is non-Archimedean, there is a nonzero nonunit $d \in R$ such that $\bigcap_{n=1}^{\infty}\left(d^{n}\right) \neq 0$. Choose $0 \neq a \in \bigcap_{n=1}^{\infty}\left(d^{n} R\right)$. Set $f=a+\sum_{n=1}^{\infty} a d^{-2^{n}} X^{n}$. Set $T=R\left[d^{-1}\right]=R_{\left\{1, d, d^{2}, \ldots\right\}}$; of course, $T$ is $R$-flat.

By the choice of $a$, we have $a d^{-2^{n}} \in R$ for each $n \geq 1$. Thus, $f \in R[[x]]$. Also, $f a^{-1}=1+\sum_{n=1}^{\infty} d^{-2^{n}} X^{n} \in T[[X]]$. In fact, since its constant term is a unit of $T, f a^{-1}$ is a unit of $T[[X]]$, and so $a=f\left(f a^{-1}\right)^{-1} \in f T[[X]]$. Therefore, $a \in f T[[X]] \cap a T[[X]]$. We shall show that

$$
a \notin(f R[[x]] \cap a R[[X]]) T[[X]] .
$$

First, we claim that if $a$ and $f$ are as above, and $r, s \in R[[X]]$ are such that $r f=s a$, then $r_{0} \in \bigcap_{n=1}^{\infty}\left(d^{n} R\right)$. To see this, observe first that equating coefficients of $X^{n}$ on each side of $r f=s a$ gives:

$$
a d^{-2^{n}} r_{0}+a d^{-2^{(n-1)}} r_{1}+\cdots+a d^{-2} r_{n-1}+a r_{n}=a s_{n}
$$

Next, multiplying both sides of this equation by $d^{2^{n-1}}$ leads to:

$$
a d^{-2^{(n-1)}} r_{0}=a\left(s_{n} d^{2^{(n-1)}}-r_{1}-\cdots-r_{n-1} d^{2^{(n-1)}-2}-r_{n} d^{2^{(n-1)}}\right) \text {. }
$$

Thus, dividing by $a$ yields:

$$
d^{-2^{(n-1)}} r_{0}=s_{n} d^{2^{(n-1)}}-r_{1}-\cdots-r_{n-1} d^{2^{(n-1)}-2}-r_{n} d^{2^{(n-1)}} \in R .
$$

This holds for all $n \geq 1$. Hence $r_{0} \in \bigcap_{n=1}^{\infty}\left(d^{n} R\right)$, as claimed.

Now suppose that $\bar{a} \in(f R[[X]] \cap a R[[X]]) T[[X]]$, contrary to what we wish to show. Then there exist $h_{i} \in f R[[X]] \cap a R[[X]]$ and $\gamma_{i} \in T[[X]]$ $(1 \leq i \leq m)$ such that $a=\sum_{i=1}^{m} h_{i} \gamma_{i}$. Furthermore, for each $i$, there are $r_{i}, s_{i} \in R[[X]]$ with $h_{i}=r_{i} f=s_{i} a$. By the above claim, $r_{i, 0} \in \bigcap_{n=1}^{\infty}\left(d^{n} R\right)$ for each $i$. Equating constant terms of $a=\sum_{i=1}^{m} h_{i} \gamma_{i}$ gives $a=\sum_{i=1}^{m} h_{i, 0} \gamma_{i, 0}$. Since $h_{i}=r_{i} f$ and $f_{0}=a$, we have $a=\sum_{i=1}^{m} r_{i, 0} a \gamma_{i, 0}$. Dividing by $a$ gives:

$$
1=\sum_{i=1}^{m} r_{i, 0} \gamma_{i, 0} \text {. }
$$

Each $\gamma_{i, 0} \in T=R\left[d^{-1}\right]$; so, for each $i$, there is an integer $u_{i}$ such that $d^{u_{i}} \gamma_{i, 0} \in R$. Let $u=\max _{1 \leq i \leq m}\left\{u_{i}\right\}$. Then $d^{u} \gamma_{i, 0} \in R$ for each $i$. Multiplying the last displayed equation by $d^{u}$ gives $d^{u}=\sum_{i=1}^{m} r_{i, 0}\left(\gamma_{i, 0} d^{u}\right)$. Since $r_{i, 0} \in$ $\bigcap_{n=1}^{\infty}\left(d^{n} R\right)$ and $\gamma_{i, 0} d^{u} \in R$ for $1 \leq i \leq m$, we have $d^{u} \in \bigcap_{n=1}^{\infty}\left(d^{n} R\right)$, whence we have $d=0$ or $d$ is a unit of $R$, contradicting our choice of $d$. Thus, $a \notin(f R[[X]] \cap a R[[X]]) T[[X]]$, and $R[[X]] \subset T[[X]]$ is not LCM-stable. 
Corollary 2.7. Let $R$ be a Prüfer domain such that $\operatorname{dim} R \geq 2$. Then there is $c \in R$ such that $R[[X]] \subset R\left[c^{-1}\right][[X]]$ is not LCM-stable.

Proof. There exists a chain of prime ideals $0 \varsubsetneqq P \varsubsetneqq M$ in $R$. Pick $c \in M \backslash P$. Since $R$ is a Prüfer domain, $R_{M}$ is a valuation domain. Therefore $P \subset c R_{M}$. Hence $P \subset \bigcap_{n=1}^{\infty}\left(c^{n} R_{M}\right)$. In particular, we may choose $0 \neq a \in\left[\bigcap_{n=1}^{\infty} c^{n} R_{M}\right] \cap$ $P$.

By the choice of $a, a c^{-n} \in R_{M}$ for each $n$. So, for each $n \geq 1$, there exist $r_{n} \in R$ and $s_{n} \in R \backslash M$ such that $a c^{-2^{n}}=r_{n} s_{n}^{-1}$. Put $f=a+\sum_{n=1}^{\infty} r_{n} X^{n} \in$ $R[[X]]$. Now $r_{n}=a s_{n} c^{-2^{n}}$, so that $f=a+\sum_{n=1}^{\infty} a s_{n} c^{-2^{n}} X^{n}$. Since $1+$ $\sum_{n=1}^{\infty} s_{n} c^{-2^{n}} X^{n}$ is a unit of $R\left[c^{-1}\right][[X]]$ and $f=a\left(1+\sum_{n=1}^{\infty} s_{n} c^{-2^{n}} X^{n}\right)$, we have $f R\left[c^{-1}\right][[X]] \cap a R\left[c^{-1}\right][[X]]=a R\left[c^{-1}\right][[X]]$. However, since $f, a \in$ $R_{M}$ [[X]] and $R_{M}$ is non-Archimedean $\left(R_{M}\right.$ is a valuation domain of dimension at least two), we may apply the proof of Proposition 2.6 to get $a \notin$ $\left(f R_{M}[[X]] \cap a R_{M}[[X]]\right) R_{M}\left[c^{-1}\right][[X]]$. (To adapt the earlier proof, get each $r_{i, 0} \in \bigcap_{n=1}^{\infty}\left(c^{n} R_{M}\right)$ since each $s_{n} \in R \backslash M$.) Since $R[[X]] \subset R_{M}[[X]]$ and $R\left[c^{-1}\right][[X]] \subset R_{M}\left[c^{-1}\right][[X]]$ we have

$$
(f R[[X]] \cap a R[[X]]) R\left[c^{-1}\right][[X]] \subset\left(f R_{M}[[X]] \cap a R_{M}[[X]]\right) R_{M}\left[c^{-1}\right][[X]] .
$$

Thus, $a \notin(f R[[X]] \cap a R[[X]]) R\left[c^{-1}\right][[X]]$, and so $R[[X]] \subset R\left[c^{-1}\right][[X]]$ is not LCM-stable.

It should be noted that the "Prüfer" hypothesis in (2.7) can be weakened to "locally divided" (in the sense of D. E. Dobbs [6]). The present formulation was chosen in order to clarify the route to our main goal, Theorem 2.11. (In this vein, we also note that the "UFD" hypothesis in Lemma 2.3 can be weakened to "GCD-domain".)

Corollary 2.7 shows that we need only consider those Prüfer domains $R$ such that $\operatorname{dim} R \leq 1$. In order to prove that the domains with the desired property are Dedekind, we shall show they are locally DVRs and Noetherian. We first consider the local case.

Theorem 2.8. Let $(V, M)$ be a rank one nondiscrete valuation domain. Then there exists a domain $D$ containing $V$ as a subring such that $V[[X]] \subset D[[X]]$ is not LCM-stable.

Proof. Since $V$ is rank one nondiscrete, there is a (surjective) valuation $v$ : q.f. $(V) \rightarrow G$ where $G$ is a dense subgroup of (the additive group of) $\mathbb{R}$. By density, we can inductively construct a sequence $\left\{\varepsilon_{j}\right\}_{j=1}^{\infty} \subset G$ such that $\lim _{j \rightarrow \infty} \varepsilon_{j}=0, \varepsilon_{j}>\varepsilon_{j+1}>0$ for all $j \geq 1$, and $\varepsilon_{j}-\varepsilon_{j+1}>\varepsilon_{j+1}-\varepsilon_{j+2}$ for all $j$. (Perhaps the easiest way to do this is to insure that $0<\varepsilon_{j+1}<2^{-1} \varepsilon_{j}$.) For each $j \geq 1$, choose $t_{j} \in V$ such that $v\left(t_{j}\right)=\varepsilon_{j}$. Put $g=\sum_{j=0}^{\infty} t_{j+1} X^{j} \in V[[X]]$. Let $Y$ be an indeterminate over $V$, and set $D=V\left[Y,\left\{Y t_{j}^{-1}\right\}_{j=2}^{\infty},\left\{Y t_{j} t_{1}^{-1}\right\}_{j=1}^{\infty}\right]$.

We claim $Y t_{1}^{-1} \notin D$. To see this, suppose, to the contrary, that $Y t_{1}^{-1} \in D$. Then $Y t_{1}^{-1}$ can be expressed as a polynomial over $V$ in $Y,\left\{Y t_{j}^{-1}\right\}_{j=2}^{\infty}$, and $\left\{Y t_{j} t_{1}^{-1}\right\}_{j=1}^{\infty}$. Working in q.f. $(V)[Y]$, we write this polynomial as $Y t_{1}^{-1}=$ $c_{0}+c_{1} Y+c_{2} Y^{2}+\cdots+c_{n} Y^{n}$, with $c_{i} \in$ q.f. $(V)$ for all $i$. Since $Y$ is an indeterminate, we have $c_{i}=0$ if $i \neq 1$. Hence any element of $D$ of the form $c_{1} Y$ (with $c_{1} \in$ q.f. $(V)$ ) must be a linear combination of $Y,\left\{Y t_{j}^{-1}\right\}_{j=2}^{\infty}$, and $\left\{Y t_{j} t_{1}^{-1}\right\}_{j=1}^{\infty}$. Thus, $Y t_{1}^{-1}=\sum_{r=1}^{a} d_{r} Y t_{j_{r}}^{-1}+\sum_{s=1}^{b} e_{s} Y t_{j_{s}} t_{1}^{-1}+c Y$ where 
$d_{r}, e_{s}, c \in V$ for all $r, s$. (Note all $j_{r} \geq 2$.) Set $u=\max _{r, s}\left\{v\left(t_{j_{r}}\right), v\left(t_{j_{s}} t_{1}^{-1}\right)\right\}$. Note that $0 \leq u<v\left(t_{1}\right)$ since $\left\{\varepsilon_{j}\right\}_{j=1}^{\infty}$ is a strictly decreasing sequence of positive numbers. Pick $t \in V$ such that $v(t)=u$. Then, multiplying the above linear combination by $t Y^{-1}$ leads to:

$$
t t_{1}^{-1}=\sum_{r=1}^{a} d_{r} t t_{j_{r}}^{-1}+\sum_{s=1}^{b} e_{s}\left(t t_{j_{s}} t_{1}^{-1}\right)+c t .
$$

By the choices of $u$ and $t$, this is an element of $V$. But $v\left(t t_{1}^{-1}\right)=v(t)-$ $v\left(t_{1}\right)<0$, whence $t t_{1}^{-1} \notin V$, a contradiction. Hence, $Y t_{1}^{-1} \notin D$, as claimed.

Consider $Y g$. Clearly, $Y g \in g D[[X]]$. Also,

$$
Y g=\sum_{j=0}^{\infty} Y t_{j+1} X^{j}=\sum_{j=0}^{\infty} t_{1}\left(Y t_{j+1} t_{1}^{-1}\right) X^{j}=t_{1} \sum_{j=0}^{\infty}\left(Y t_{j+1} t_{1}^{-1}\right) X^{j} \in t_{1} D[[X]] .
$$

Hence, $Y g \in g D[[X]] \cap t_{1} D[[X]]$. We shall show that

$$
Y g \notin\left(g V[[X]] \cap t_{1} V[[X]]\right) D[[X]],
$$

and therefore that $V[[X]] \subset D[[X]]$ is not LCM-stable.

Suppose $f \in g V[[X]] \cap t_{1} D[[X]]$. Then there exist $r, s \in V[[X]]$ with $f=g r=t_{1} s$. We can now apply [1, Lemma, p. 191] which seems tailor-made for such series. The result is $v\left(r_{i}\right) \geq v\left(t_{1}\right)-v\left(t_{j+1}\right)$ for each $i$ and for each $j$. Since $\lim _{j \rightarrow \infty} v\left(t_{j+1}\right)=0$, we have $v\left(r_{i}\right) \geq v\left(t_{1}\right)$ for each $i$. Thus $r_{i} t_{1}^{-1} \in V$ for each $i$, so that $r t_{1}^{-1} \in V[[X]]$. Next, write $f=g r=g t_{1}\left(r t_{1}^{-1}\right)$ and equate constant terms. Since $g_{0}=t_{1}$, this gives $f_{0}=g_{0} t_{1}\left(r_{0} t_{1}^{-1}\right)=t_{1}^{2} r_{0} t_{1}^{-1}=t_{1} r_{0}$. Thus, $v\left(f_{0}\right)=v\left(t_{1} r_{0}\right)=v\left(t_{1}\right)+v\left(r_{0}\right) \geq v\left(t_{1}\right)+v\left(t_{1}\right)=v\left(t_{1}^{2}\right)$. Hence, there is $f_{0}^{*} \in V$ such that $f_{0}=t_{1}^{2} f_{0}^{*}$.

Now, if $Y g \in\left(g V[[X]] \cap t_{1} V[[X]]\right) D[[X]]$, then $Y g=\sum_{k=1}^{m} f_{k} \alpha_{k}$ for some $f_{k} \in g V[[X]] \cap t_{1} V[[X]]$ and $\alpha_{k} \in D[[X]]$. Equating constant terms and using the result of the preceding paragraph give $f_{k, 0}^{*} \in V$ (for $1 \leq k \leq m$ ) such that $Y g_{0}=Y t_{1}=\sum_{k=1}^{m} f_{k, 0} \alpha_{k, 0}=\sum_{k=1}^{m}\left(t_{1}^{2} f_{k, 0}^{*}\right) \alpha_{k, 0}=t_{1}^{2} \sum_{k=1}^{m} f_{k, 0}^{*} \alpha_{k, 0}$. Thus, $Y t_{1}=t_{1}^{2} \sum_{k=1}^{m} f_{k, 0}^{*} \alpha_{k, 0}$. Dividing by $t_{1}^{2}$ yields $Y t_{1}^{-1}=\sum_{k=1}^{m} f_{k, 0}^{*} \alpha_{k, 0} \in D$. But this contradicts the claim that was established above. Hence, $Y g \notin$ $\left(g V[[X]] \cap t_{1} V[[X]]\right) D[[X]]$ and $V[[X]] \subset D[[X]]$ is not LCM-stable.

The local case that was considered in Theorem 2.8 is next used to eliminate any one-dimensional Prüfer domain which is not almost Dedekind. (As in [10], a domain $R$ is said to be almost Dedekind if $R_{M}$ is a DVR for each maximal ideal $M$ of $R$. Each almost Dedekind domain is Prüfer and of dimension at most one. The converse is false.)

Corollary 2.9. Let $R$ be a Prüfer domain. If $\operatorname{dim} R=1$ and $R$ is not almost Dedekind, then there is a domain $D$ containing $R$ as a subring such that $R[[X]] \subset D[[X]]$ is not LCM-stable.

Proof. Since $R$ is a Prüfer domain, $R_{M}$ is a valuation domain for each prime ideal $M$ of $R$. Moreover, $\operatorname{dim} R=1$ shows $R_{M}$ is a rank one valuation domain for each nonzero prime ideal $M$. Also, since $R$ is not almost Dedekind, there is at least one prime ideal $M$ such that $R_{M}$ is nondiscrete. Fix a prime $M$ such that $R_{M}$ is a nondiscrete valuation domain. 
As in the proof of Theorem 2.8, choose $t_{j} \in R_{M}$ such that $v\left(t_{j}\right)=\varepsilon_{j}$ for all $j \geq 1$, where the sequence $\left\{\varepsilon_{j}\right\}_{j=1}^{\infty}$ is as in the earlier proof. Now, for each $j$, write $t_{j}=r_{j} s_{j}^{-1}$, with $r_{j} \in R, s_{j} \in R \backslash M$. Since $s_{j}$ is a unit of $R_{M}$, we have $v\left(s_{j}\right)=0$ for $j \geq 1$. Thus $v\left(t_{j}\right)=v\left(r_{j} s_{j}^{-1}\right)=v\left(r_{j}\right)-v\left(s_{j}\right)=v\left(r_{j}\right)$ for all $j \geq 1$. So, by putting $g=\sum_{j=0}^{\infty} r_{j+1} X^{j}$, the proof of Theorem 2.8 shows there exists a domain $D$ containing $R_{M}$ (and hence $R$ ) as a subring such that $g D[[X]] \cap r_{1} D[[X]] \supsetneqq\left(g R_{M}[[X]] \cap r_{1} R_{M}[[X]]\right) D[[X]]$. But $g, r_{1} \in R[[X]]$ and $g R_{M}[[X]] \cap r_{1} R_{M}[[X]] \supset g R[[X]] \cap r_{1} R[[X]]$. Thus, $g D[[X]] \cap r_{1} D[[X]] \supsetneqq$ $\left(g R[[X]] \cap r_{1} R[[X]]\right) D[[X]]$, and so the domain $D$ has the required properties.

Finally, we show the almost Dedekind, but not Dedekind, domains do not have the desired property. It is convenient to recall that a domain $R$ is an $S F T$-ring if, for each ideal $I$ of $R$, there is a finitely generated ideal $J$ of $R$ such that $J \subset I$ and there exists an integer $k \geq 1$ such that $i^{k} \in J$ for each $i \in I$.

Theorem 2.10. Let $R$ be an almost Dedekind, but not Dedekind, domain. Then there exists a domain $D$ containing $R$ as a subring such that $R[[X]] \subset D[[X]]$ is not LCM-stable; it can be arranged that $D$ is an overring of $R$.

Proof. By a remark of Arnold [3, $\S 3$, p. 4], $R$ is not an SFT ring. Hence, by [2, Theorem 1], there exists a prime ideal $P$ of $R$ such that $P[[X]] \neq$ $\operatorname{rad}(P R[[X]])$. Then [4, Proposition 1] shows height $(P[[X]]) \geq 2$. We claim $R[[X]]_{P[X]]}$ is not a valuation domain; indeed, since $\operatorname{dim}\left(R[[X]]_{P[[X]]}\right) \geq 2$, $R[[X]]_{P[[X]]}$ is not a DVR, and so the claim follows from [4, Theorem 1]. Since $R_{P}$ is a DVR and $R[[X]]_{P[X]]}$ is not a valuation domain, [4, Theorem 3] shows $R_{P}[[X]] \cap$ q.f. $(R[[X]]) \not \subset R[[X]]_{P[[X]]}$.

Pick $\gamma \in R_{P}[[X]] \cap$ q.f. $(R[[X]]) \backslash R[[X]]_{P[[X]]}$. Since $\gamma \in$ q.f. $(R[[X]])$, we may write $\gamma=a b^{-1}$ with $0 \neq a, b \in R[[X]]$. Thus $a=b \gamma$ and hence $a R_{P}[[X]] \cap b R_{P}[[X]]=a R_{P}[[X]]=a R_{P}[[X]]$. We shall show if $R[[X]] \subset$ $R_{P}[[X]]$ is LCM-stable, then $\gamma \in R[[X]]_{P[[X]]}$, contradicting the choice of $\gamma$.

Now, if we assume $R[[X]] \subset R_{P}[[X]]$ is LCM-stable then

$$
a \in(a R[[X]] \cap b R[[X]]) R_{P}[[X]] .
$$

Hence, there exist $h_{i} \in a R[[X]] \cap b R[[X]]$ and $\tau_{i} \in R_{P}[[X]]$ (for $1 \leq i \leq n$ ) such that $a=\sum_{i=1}^{n} h_{i} \tau_{i}$. Also, there exist $r_{i}, s_{i} \in R[[X]]$ (for $1 \leq i \leq n$ ) with $h_{i}=a r_{i}=b s_{i}$. Thus $a=\sum_{i=1}^{n} h_{i} \tau_{i}=\sum_{i=1}^{n}\left(a r_{i}\right) \tau_{i}=a \sum_{i=1}^{n} r_{i} \tau_{i}$. Therefore $1=\sum_{i=1}^{n} r_{i} t_{i}$. Since $R_{P}[[X]]$ is a local ring, there exists $i_{0}$ such that $r_{i_{0}} t_{i_{0}}$ is a unit in $R_{P}[[X]]$. Without loss of generality, $i_{0}=1$. But then $r_{1}$ is a unit in $R_{P}[[X]]$ and hence $r_{1,0} \in R \backslash P$. Since $r_{1} \in R[[X]], r_{1}$ is a unit in $R[[X]]_{P[[X]]}$. Thus, $a r_{1}=b s_{1}$ leads to $\gamma=a b^{-1}=s_{1} r_{1}^{-1} \in R[[X]]_{P[[X]]}$, the desired contradiction. Hence $R[[X]] \subset R_{P}[[X]]$ is not LCM-stable and $D=R_{P}$ satisfies the conclusion of the theorem.

Hence, (2.1)-(2.10) combine to establish our main result.

Theorem 2.11. A domain $R$ is a Dedekind domain if and only if $R[[X]] \subset$ $T[[X]]$ is LCM-stable for all domains $T$ containing $R$ as a subring.

The question whether the domains $T$ in Theorem 2.11 can be restricted to be overrings of $R$ is open. By our methods of proof, one need only settle this issue in the context of Theorem 2.8. 
Remark 2.12 sketches some related work on flatness. First, we give some relevant background.

In [7], Dobbs introduced the notion of $n$-flatness, where $n$ is a positive integer. It is classical [5] that an $R$-module $M$ is $R$-flat if and only if $M$ is $n$-flat over $R$ for each integer $n$. Dobbs [8, Theorem 3.3] shows that LCMstability is equivalent to 2-flatness. Hence, Theorem 2.11 may be rephrased as follows. The domain $R$ is a Dedekind domain if and only if $T[[X]]$ is 2 -flat over $R[[X]]$ for each domain $T$ containing $R$ as a subring. Replacing "2-flat" with "flat", we feel it is natural to ask which domains have the property $(*)$ that $T[[x]]$ is a flat $R[[X]]$-module for all domains $T$ containing $R$ as a subring. In view of the above remarks, Theorem 2.11 shows that any domain having the property $(*)$ must be a Dedekind domain. That some domain does in fact have property $(*)$ is included in Remark $2.12(\mathrm{a})$.

Remark 2.12. (a) If $A$ is a subring of the domain $B$ such that q.f. $(A) \subset$ $B$, then $B[[X]]$ is $A[[X]]-$ flat. (The proof is a lengthy calculation using the characterization of flatness in terms of conductors [5, Exercise 2.2, p. 47].)

(b) One motivation for (a) is the trivium that fields satisfy $(*)$. In fact, by using [5, Exercise 17, p. 250$]$, we see that $B\left[\left[X_{1}, \ldots, X_{n}\right]\right]$ is $K\left[\left[X_{1}, \ldots, X_{n}\right]\right]-$ flat for all $n \geq 1$ whenever $B$ contains the field $K$ as a subring. We next state a result leading to a partial result involving arbitrary Dedekind domains: If $R$ and $T$ are Noetherian domains such that $R$ is a subring of $T$ and $T$ is $R$-flat, then $T[[X]]$ is $R[[X]]$-flat. (The proof applies [5, Theorem 3, p. 203] to completion in the $X$-adic topology, to see that $T[[X]]$ is $T[X]$-flat; and it concludes via transitivity of flatness and [5, Proposition 4, p. 231].)

(c) If $R$ is Dedekind, each domain containing $R$ as a subring is $R$-flat. Hence (b) yields: if $R$ is a Dedekind domain, then $T[[X]]$ is $R[[X]]-$ flat for each Noetherian domain $T$ containing $R$ as a subring.

The question of characterizing domains having property $(*)$ now reduces to determining whether "Noetherian" may be deleted from the preceding statement.

\section{REFERENCES}

1. M. Anderson and J. Watkins, Coherence of power series rings, J. Algebra 107 (1987), 187-194.

2. J. T. Arnold, Krull dimension in power series rings, Trans. Amer. Math. Soc. 177 (1973), 299-304.

3. __ Power series rings over Prüfer domains, Pacific J. Math. 44 (1973), 1-11.

4. J. T. Arnold and J. Brewer, When $(D[[X]])_{P[[X]]}$ is a valuation ring, Proc. Amer. Math. Soc. 37 (1973), 326-332.

5. N. Bourbaki, Commutative algebra, Addison-Wesley, Reading, MA, 1972.

6. D. E. Dobbs, On locally divided integral domains and CPI-overrings, Internat. J. Math. Math. Sci. 4 (1981), 119-135.

7. _ On n-flat modules over a commutative ring, Bull. Austral. Math. Soc. 43 (1991), 491-498.

8. _ On the criteria of D. D. Anderson for invertible and flat ideals, Canad. Math. Bull. 29 (1986), 25-32.

9. R. Gilmer, Finite element factorization in group rings, Lecture Notes in Pure and Appl. Math., vol. 7, Dekker, New York, 1974.

10. 
11. I. Kaplansky, Commutative rings, 2nd ed., Univ. of Chicago Press, Chicago and London, 1974.

12. F. Richman, Generalized quotient rings, Proc. Amer. Math. Soc. 16 (1965), 794-799.

13. J. Sato and K. Yoshida, The LCM-stability on polynomial extensions, Math. Rep. Toyama Univ. 10 (1987), 75-84.

14. P. Sheldon, How changing $D[[X]]$ changes its quotient field, Trans. Amer. Math. Soc. 159 (1971), 223-244.

15. H. Uda, LCM-stableness in ring extensions, Hiroshima Math. J. 13 (1983), 357-377.

16. _,$G_{2}$-stableness and LCM-stableness, Hiroshima Math. J. 18 (1988), 47-52.

Department of Mathematics, University of Tennessee, Knoxville, Tennessee 379961300

Current address: 748 Jamie Way, Woodstock, Georgia 30188 